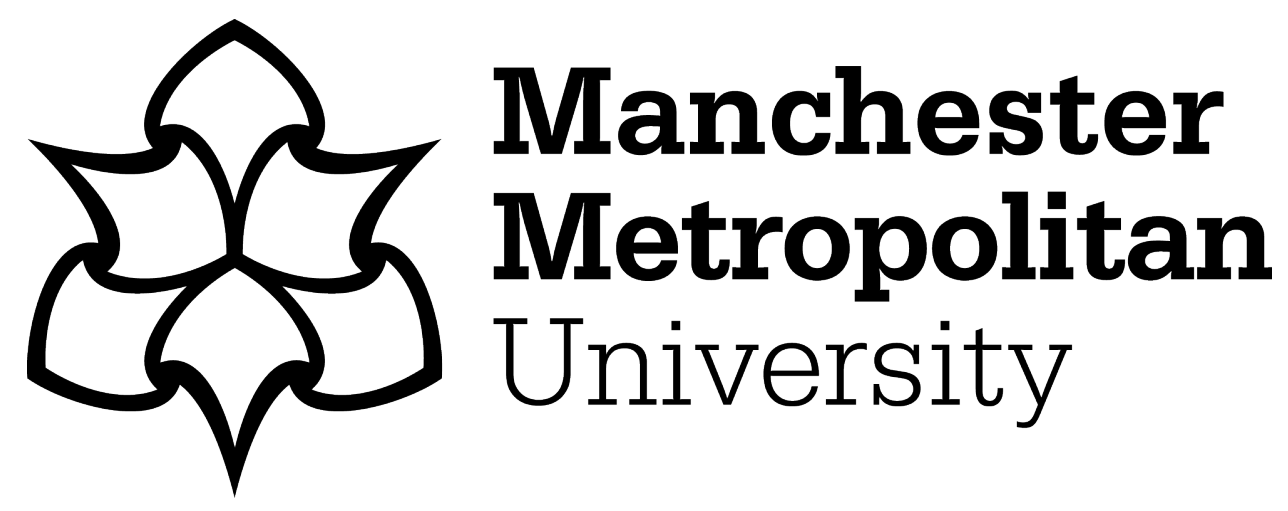

Redfern, J ORCID logoORCID: https://orcid.org/0000-0003-0958-683X, Wallace, J, van Belkum, A, Jaillard, M, Whittard, E, Ragupathy, R ORCID logoORCID: https://orcid.org/0000-0002-7199-5020, Verran, J ORCID logoORCID: https://orcid.org/0000-0002-5539-6896, Kelly, P ORCID logoORCID: https://orcid.org/0000-0003-1008-4941 and Enright, MC (2021) Biofilm associated genotypes of multiple antibiotic resistant Pseudomonas aeruginosa. BMC Genomics, 22. p. 572. ISSN 1471-2164

Downloaded from: https://e-space.mmu.ac.uk/628284/

Version: Published Version

Publisher: BioMed Central

DOI: https://doi.org/10.1186/s12864-021-07818-5

Usage rights: Creative Commons: Attribution 4.0

Please cite the published version 


\title{
Biofilm associated genotypes of multiple antibiotic resistant Pseudomonas aeruginosa
}

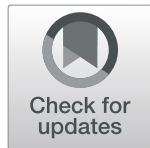

James Redfern', Janine Wallace ${ }^{1}$, Alex van Belkum², Magali Jaillard², Elliot Whittard ${ }^{1}$, Roobinidevi Ragupathy', Joanna Verran', Peter Kelly ${ }^{1}$ and Mark Charles Enright ${ }^{*}$

\begin{abstract}
Background: Pseudomonas aeruginosa is a ubiquitous environmental microorganism and also a common cause of infection. Its ability to survive in many different environments and persistently colonize humans is linked to its presence in biofilms formed on indwelling device surfaces. Biofilm promotes adhesion to, and survival on surfaces, protects from desiccation and the actions of antibiotics and disinfectants.

Results: We examined the genetic basis for biofilm production on polystyrene at room $\left(22^{\circ} \mathrm{C}\right)$ and body temperature $\left(37^{\circ} \mathrm{C}\right.$ ) within $280 \mathrm{P}$. aeruginosa. 193 isolates (69\%) produced more biofilm at $22^{\circ} \mathrm{C}$ than at $37^{\circ} \mathrm{C}$. Using GWAS and pan-GWAS, we found a number of accessory genes significantly associated with greater biofilm production at $22{ }^{\circ} \mathrm{C}$. Many of these are present on a $165 \mathrm{~kb}$ region containing genes for heavy metal resistance (arsenic, copper, mercury and cadmium), transcriptional regulators and methytransferases. We also discovered multiple core genome SNPs in the A-type flagellin gene and Type II secretion system gene xpsD. Analysis of biofilm production of isolates of the MDR ST111 and ST235 lineages on stainless-steel revealed several accessory genes associated with enhanced biofilm production. These include a putative translocase with homology to a Helicobacter pylori type IV secretion system protein, a TA system II toxin gene and the alginate biosynthesis gene algA, several transcriptional regulators and methytransferases as well as core SNPs in genes involved in quorum sensing and protein translocation.
\end{abstract}

Conclusions: Using genetic association approaches we discovered a number of accessory genes and core-genome SNPs that were associated with enhanced early biofilm formation at $22^{\circ} \mathrm{C}$ compared to $37^{\circ} \mathrm{C}$. These included a $165 \mathrm{~kb}$ genomic island containing multiple heavy metal resistance genes, transcriptional regulators and methyltransferases. We hypothesize that this genomic island may be associated with overall genotypes that are environmentally adapted to survive at lower temperatures. Further work to examine their importance in, for example gene-knockout studies, are required to confirm their relevance. GWAS and pan-GWAS approaches have great potential as a first step in examining the genetic basis of novel bacterial phenotypes.

Keywords: Pseudomonas aeruginosa, Genomics, GWAS, Biofilm

\footnotetext{
* Correspondence: m.enright@mmu.ac.uk

${ }^{1}$ Faculty of Science and Engineering, Manchester Metropolitan University, Chester Street, Manchester, United Kingdom

Full list of author information is available at the end of the article
}

(c) The Author(s). 2021 Open Access This article is licensed under a Creative Commons Attribution 4.0 International License, which permits use, sharing, adaptation, distribution and reproduction in any medium or format, as long as you give appropriate credit to the original author(s) and the source, provide a link to the Creative Commons licence, and indicate if changes were made. The images or other third party material in this article are included in the article's Creative Commons licence, unless indicated otherwise in a credit line to the material. If material is not included in the article's Creative Commons licence and your intended use is not permitted by statutory regulation or exceeds the permitted use, you will need to obtain permission directly from the copyright holder. To view a copy of this licence, visit http://creativecommons.org/licenses/by/4.0/ The Creative Commons Public Domain Dedication waiver (http://creativecommons.org/publicdomain/zero/1.0/) applies to the data made available in this article, unless otherwise stated in a credit line to the data. 


\section{Background}

Pseudomonas aeruginosa is a mono-flagellate, Gramnegative bacterium that is present in most environments. A frequent coloniser of humans, other animals and plants, $P$. aeruginosa is also a very common opportunistic pathogen able to grow at a variety of temperatures [1]. The species is a leading cause of severe, lifethreatening nosocomial human infections and the major pathogen associated with lung infections of patients with cystic fibrosis. This microorganism has been classified as one of the major species associated with multiple antimicrobial resistance of urgent public health concern by the Infectious Diseases Society of America [2], Centers for Disease Control and Prevention [3] and the World Health Organization [4].

A variety of typing methods including multilocus sequence typing (MLST) [5] and more recently, genomic sequencing studies [6-9] have shown that $P$. aeruginosa has a non-clonal, epidemic population structure in which successful clones occasionally arise and are globally transmitted [10]. Multidrug resistant (MDR) isolates of lineages associated with outbreaks of infection belonging to MLST Sequence Types (STs) 111 and ST235 are two such 'high risk' clones that have global distributions and numerous and transferable antibiotic resistances [11]. Isolates of these lineages contain a large number of horizontally transferred $\beta$-lactamase genes [8] and are highly virulent in comparison to a third, less significant lineage - ST175 [12].

$P$. aeruginosa has many phenotypic features that promote its success as a pathogen. In common with other bacterial pathogens $P$. aeruginosa usually exists within biofilms. These are complex microbial communities associated with extracellular polymeric matrices that help bacteria resist desiccation, mechanical removal and the actions of antibiotics and disinfectants [13]. In human tissue, biofilm-associated $P$. aeruginosa are difficult to eradicate and represent infectious foci that can lead to serious systemic disease. P. aeruginosa biofilms are found at surface-liquid, surface-air and liquid-air interfaces, and are a significant clinical problem in wounds and the cystic fibrosis lung [14]. Polymicrobial biofilms are also recognized as important niches for MDR evolution, as they represent a significant reservoir for horizontal gene transfer within and between bacterial species [15].

In addition to comprehending in vivo biofilm formation, understanding $P$. aeruginosa biofilm development and persistence on other surfaces is required. Stainlesssteel is the most common surface material used in many industries where control of microorganisms is important, including healthcare. Properties of stainless-steel include resistance to corrosion, easiness to clean and a level of hardness likely to limit scratches and other defects [16]. However, many of these surfaces have been shown to allow the formation of biofilms, for example in hard-to-clean locations, and those with a favourable environment, such as sinks and pipes [17]. ST111 isolates have been shown to produce significantly more biofilm compared to those from non-high-risk clones [18] whilst high-risk clones are over-represented in locations such as hospital sink pipes [19]. A recent study strongly implicates the presence of $P$. aeruginosa biofilm in hospital waste-water pipes as being responsible for an outbreak of infection due to MDR ST111 and ST235 in a haematology unit [20] and policies promoting the replacement of sink units to reduce such sources of infection have been introduced in the United Kingdom [21].

There is a need to better understand the clinical importance of biofilms in hospital environments starting with how biofilm production changes when $P$. aeruginosa cells move from the hospital environment to the human body. Pathogen genomics is becoming a key tool for epidemiological surveillance of many species and can be used to guide clinical treatment and outbreak control $[22,23]$. The large number of pathogen genomes and associated metadata in the public domain represents a rich and growing resource for bioinformatic investigations of pathogenicity, antibiotic resistance, vaccine susceptibility and other important phenotypes. Genome Wide Association Studies (GWAS) have been used to great effect in identifying genetic determinants contributing to disease pathology in human medicine. Thus far, microbial GWAS and more generic Whole Genome Sequencing (WGS) studies have focused on the molecular epidemiology of infectious disease and particularly on associations between the genome and antimicrobial resistance and / or pathogenicity. There is huge potential for such methods to help understand the genetic basis of other phenotypes, such as key processes that are important in microbial ecology or industrial microbiology [24, 25].

In this study we sought to find genes and core genome SNPs associated with biofilm production by examining associations within the $P$. aeruginosa pan-genome and biofilm-producing phenotypes in two experiments using a collection of $280 P$. aeruginosa isolates whose genomes were sequenced in a previous study [6]. In the first we measured the density of biofilm produced by $P$. aeruginosa grown in polystyrene microtiter plates at ambient $\left(22^{\circ} \mathrm{C}\right)$ and body $\left(37^{\circ} \mathrm{C}\right)$ temperatures. In the second we measured the biofilm density of isolates from this larger collection belonging to the international MDR ST111 and ST235 lineages grown on stainless-steel.

\section{Results}

Temperature-dependent biofilm analysis

Biofilm formation varied greatly between isolates at both 22 and $37{ }^{\circ} \mathrm{C}$ (Fig. 1). Of the 280 isolates studied, $69 \%$ 


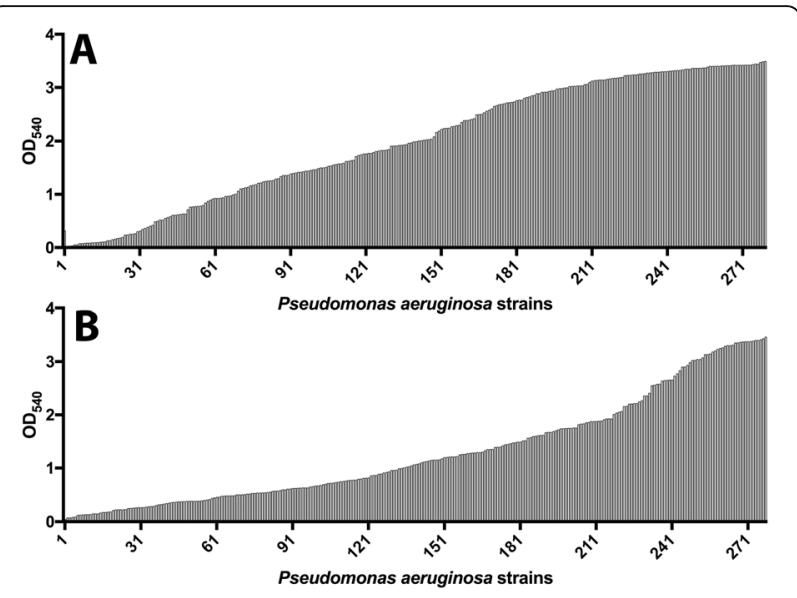

Fig. 1 Optical density related to biofilm stained with $0.1 \%$ crystal violet and solubilised in $30 \%$ acetic acid at either $22{ }^{\circ} \mathrm{C}$ (A) or $37^{\circ} \mathrm{C}$ (B) for 280 Pseudomonas aeruginosa isolates. $n=4$ for each isolate/temperature condition

( $n=193)$ produced more biofilm at $22{ }^{\circ} \mathrm{C}$ (mean $\mathrm{OD}_{540}$ of 1.98) compared to $37{ }^{\circ} \mathrm{C}$ (mean $\mathrm{OD}_{540}$ measurement of 1.29). Isolates that produced more biofilm at $22{ }^{\circ} \mathrm{C}$ than at $37{ }^{\circ} \mathrm{C}$ had a mean $\mathrm{OD}_{540}$ measure of 2.35 at $22{ }^{\circ} \mathrm{C}$ and 0.97 at $37{ }^{\circ} \mathrm{C}$. Isolates producing more biofilm at $37^{\circ} \mathrm{C}$ had a mean $\mathrm{OD}_{540}$ measure of 1.16 at $22{ }^{\circ} \mathrm{C}$ and 2.02 at $37{ }^{\circ} \mathrm{C}$. Isolates producing more biofilm at $22{ }^{\circ} \mathrm{C}$ belonged to 84 different MLST STs compared to 61 of isolates producing more biofilm at $37{ }^{\circ} \mathrm{C}$. Isolates demonstrated more variation in OD540 measures when grown at $37{ }^{\circ} \mathrm{C}($ mean $=1.291$, standard deviation $=0.98$, coefficient of variation $=76.12 \%$ ), than at $22{ }^{\circ} \mathrm{C}$ (mean of 1.983 , standard deviation of 1.12, coefficient of

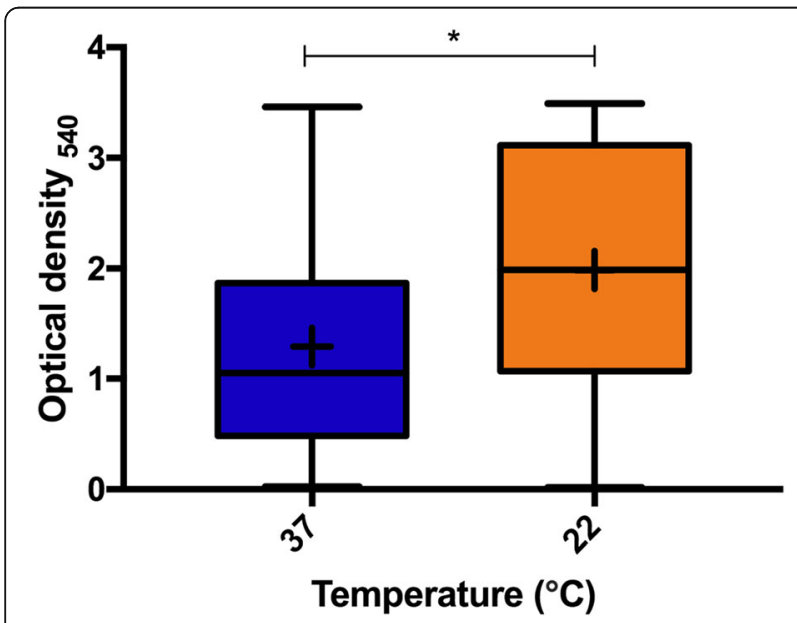

Fig. 2 Min and max box plot demonstrating the variation in biofilm formation for 280 Pseudomonas aeruginosa isolates at two different temperatures as measured by optical density. Average values are denoted by + . Significance $(P<0.05)$, as assessed by T-Test is denoted by *. Each box plot represents data for 280 isolates variation $=56.34 \%)$. (Fig. 2). Increased biofilm growth phenotype at $37{ }^{\circ} \mathrm{C}$ was widely distributed across the phylogeny as shown in Fig. 3.

Scoary analyses yielded 2,834 clusters of orthologous groups (COGs) and genes associated with the phenotypic trait of producing more biofilm at $22{ }^{\circ} \mathrm{C}$ compared to $37^{\circ} \mathrm{C}$ (naïve $p<0.05$ ) (Suppl. Table 3). None of these hits were significant using a Bonferroni corrected $p$ value of $<0.05$ but 74 were significantly associated with this phenotype using the conservative, but less stringent, Benjamini-Hochberg correction at $p<0.05$. Pyseer analysis using the same input gave 1,015 significant hits (naïve $p<0.05$ ) and 155 using a lrt (likelihood - ratio test) $p<0.01$ (Suppl. Table 4). The top 20 most significant hits using the two tests are shown in Table 1 with the 14 genes present in both lists shown in bold type. Scoary hits were ordered by Benjamini-Hochberg - adjusted $p$-values and Pyseer hits by lrt- $p$-values. All hits are shown in Suppl. Tables 3 and 4. 20/20 of the most significant hits identified by Scoary are present in the genome of isolate WH-SGI-V-07050 as are 15 / 20 using Pyseer and genes in Table 1 were therefore numbered according to this genome (when present). These genes include acr3, ars $C$, ars $R$ and $\operatorname{arsH}$ - four genes involved in arsenic resistance / reduction as well as two methyltransferases and an integrating conjugative element (ICE) protein and an ICE relaxase. The gene ars $R$-family transcriptional regulators are considered to be important in many physiological processes, including biofilm production [27]. Presence of arsenic in bacterial cells has been demonstrated to affect biofilm synthesis as well as chemotaxis and motility [28], and there is a suggestion that arsenic can prevent the switch between planktonic and sessile lifestyle [29]. The presence of the flagellar protein $\mathrm{FliC}$ is also significantly associated with increased biofilm growth at $22{ }^{\circ} \mathrm{C}$ compared to $37{ }^{\circ} \mathrm{C}$ Pyseer (but not Scoary). Flagellar motility is wellknown as a requirement for biofilm production in $P$. aeruginosa[30] and suppression of $f l i C$ has been linked to increasing temperature in $P$. syringae[31], with similar temperature-association of flagellar being reported in Listeria monocytogenes and Proteus vulgaris[32, 33]. The gene $c l s A$, a cardiolipin synthase has demonstrated impact on biofilm formation in E. coli [34] although association with temperature has not been evaluated. The two methyltransferases and in particular the SAM (S-adenosylmethionine) - dependent methyltransferase may have a role in $\mathrm{N}$-acyl-homoserine lactone synthesis, the key molecules in $P$. aeruginosa quorum sensing (QS) [35]. QS has long been known to play a central role in biofilm formation in $P$. aeruginosa [36] however further work would be required to establish the importance of these methyltransferases in QS and biofilm production. 


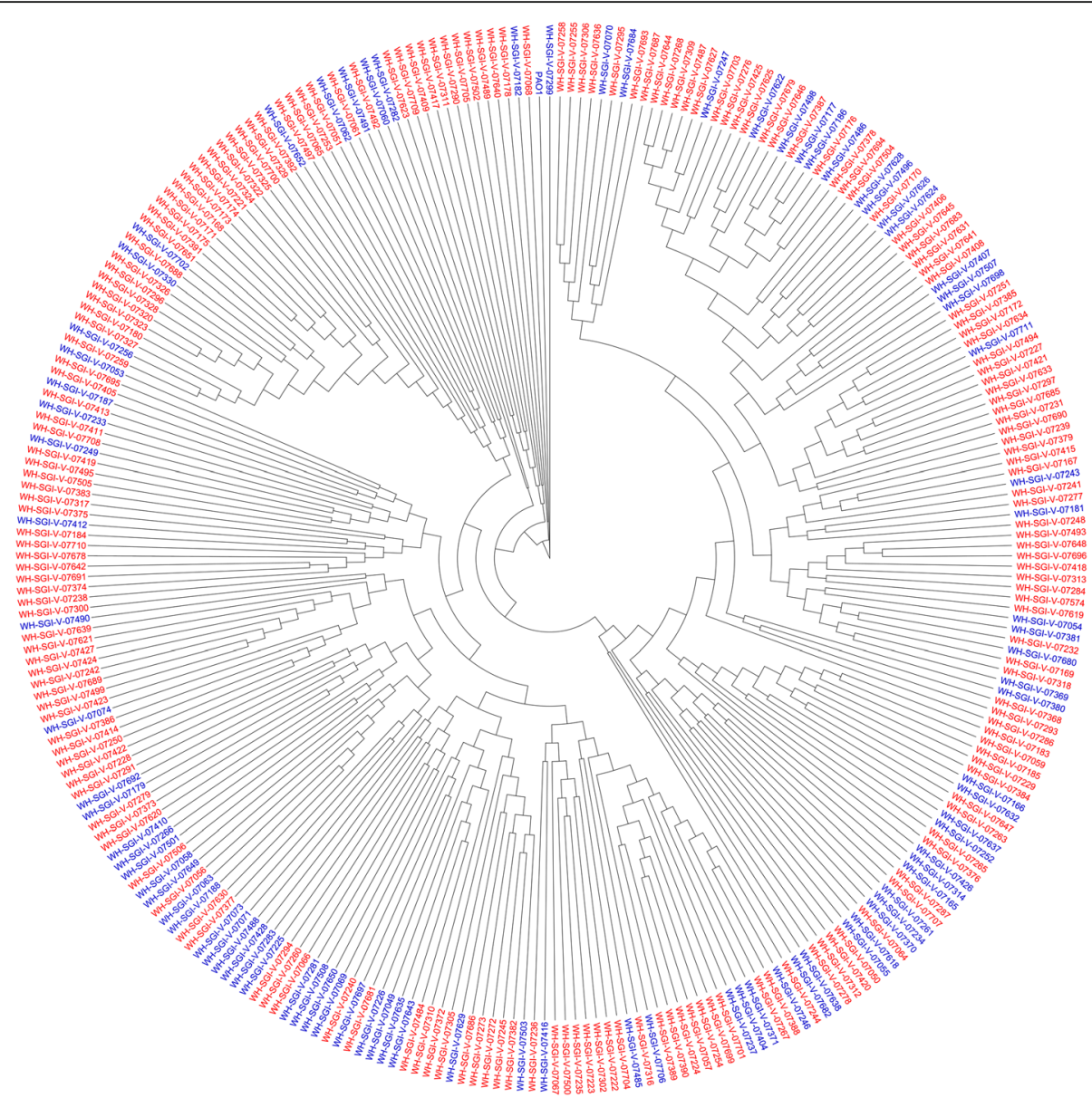

Fig. 3 Unrooted maximum parsimony (MP) tree based on core genome SNPs of 280 Pseudomonas aeruginosa isolates. Tree is a consensus of 100 MP trees generated using kSNP3 [26]. Names highlighted red represent an isolate that produced more biofilm at $22^{\circ} \mathrm{C}$ and names highlighted blue represent an isolate that produced more biofilm at $37^{\circ} \mathrm{C}$

The majority $(20 / 26)$ of the most significant hits presented in Table 1 are in close proximity to each other in the WH-SGI-V-07050 chromosome - genes numbered 373 (WH-SGI-V-07050_00373 in Table 1) to 461 (WHSGI-V-07050_00461). These genes are present in between 63 and 80 of the 280 study isolates. Comparative analysis of DNA in this region from the genome of isolate WH-SGI-V-07050 with other study isolate genomes show that this region is $165,376 \mathrm{bp}$ long and contains 149 genes starting from WH-SGI-V-07050_349 to WHSGI-V-07050_498. A Blastn search of this region showed $>99 \%$ DNA similarity over the entire region with several finished genomic sequences including strain FDAARGOS-532 (GenBank accession GI:1519006927). A manual alignment of this genome with those of $\mathrm{WH}$ SGI-V-07050 and PAO1 (GI:110645304) was performed using Artemis Comparison Tool [37] (Fig. 4). This shows that this region is not present in strain PAO1 except for a region of 13,273 bp corresponding to bases 2,923,150 to $2,936,423$ of PAO1 common to all three genomes.
This conserved region contains ten open reading frames including pgsA at the 5' end and the hypothetical protein gene PAO1_02672 at the 3' end.

We used Phandango to visualise the genomes of the 280 isolates in this study for the presence (or absence) of genes corresponding to WH-SGI-V-07050_349 to WHSGI-V-07050_498. Figure 4 shows that most of these genes are present in the genomes of many isolates and that their distribution is not strongly associated with core genome phylogeny although only a small number of genomes share the total length of this region with WH-SGI-V-07050 (shown in blue in Fig. 4). Besides containing a cluster of arsenic resistance genes (see above) this region also contains genes encoding heavy metal-resistance genes for copper (copA2, copA_3, copA 4, copB_1, copC, copD, copK), mercury (merR_1, merP 1 ), and cadmium (cadA_1) that (with the exception of $\operatorname{cadA}$ ) are significantly associated with the phenotype of producing more biofilm at ambient compared to body temperature (Scoary and Pyseer ranked hits are shown 
Table 1 Ranked list of the most significant genes / COGs associated with greater biofilm growth at $22^{\circ} \mathrm{C}$ than $37^{\circ} \mathrm{C}$ using Scoary and Pyseer SCOARY

\begin{tabular}{|c|c|c|c|c|c|c|}
\hline & COG / Gene & Function & Presence & OR & Ben $p$ & Example (isolate_gene position) \\
\hline 1 & group_8321 & Methyltransferase & 68 & 6.389 & 0.018 & WH-SGI-V-07050_00375 \\
\hline 2 & group_15072 & Hypothetical & 80 & 5.044 & 0.018 & WH-SGI-V-07050_00461 \\
\hline 3 & group_1519 & SAM-dependent methyltransferase & 79 & 4.932 & 0.018 & WH-SGI-V-07050_00374 \\
\hline 4 & group_15041 & Integrating conjugative element protein & 75 & 5.251 & 0.018 & WH-SGI-V-07050_00387 \\
\hline 5 & $\begin{array}{l}\text { group_11043 } \\
\text { (arsC_1) }\end{array}$ & Arsenate reductase (glutaredoxin) & 78 & 4.823 & 0.018 & WH-SGI-V-07050_00379 \\
\hline 6 & acr3 & ACR3 family arsenite efflux transporter & 78 & 4.823 & 0.018 & WH-SGI-V-07050_00380 \\
\hline 7 & arsC_2 & Arsenate reductase & 78 & 4.823 & 0.018 & WH-SGI-V-07050_00381 \\
\hline 8 & group_11046 & ArsR family transcriptional regulator & 78 & 4.823 & 0.018 & WH-SGI-V-07050_00382 \\
\hline 9 & group_2314 & Hypothetical & 71 & 4.823 & 0.018 & WH-SGI-V-07050_00373 \\
\hline 10 & arsH_1 & Arsenical resistance protein & 78 & 4.823 & 0.018 & WH-SGI-V-07050_00378 \\
\hline 11 & ydfG & NADP-dependent 3-hydroxy acid dehydrogenase & 73 & 5.015 & 0.018 & WH-SGI-V-07050_00440 \\
\hline 12 & group_15062 & TetR/AcrR family transcriptional regulator & 73 & 5.015 & 0.018 & WH-SGI-V-07050_00441 \\
\hline 13 & group_11053 & Integrating conjugative element relaxase, $\mathrm{PFGI}-1$ class & 77 & 4.715 & 0.018 & WH-SGI-V-07050_00417 \\
\hline 14 & qorB & Quinone oxidoreductase & 77 & 4.715 & 0.018 & WH-SGI-V-07050_00419 \\
\hline 15 & group_832 & Hypothetical & 77 & 4.715 & 0.018 & WH-SGI-V-07050_00384 \\
\hline 16 & group_11042 & Hypothetical & 63 & 5.658 & 0.018 & WH-SGI-V-07050_00376 \\
\hline 17 & group_15038 & Integrating conjugative element protein pill, pfgi-1 & 76 & 4.608 & 0.018 & WH-SGI-V-07050_00383 \\
\hline 18 & group_15039 & TIGR03759 family integrating conjugative element protein & 76 & 4.608 & 0.018 & WH-SGI-V-07050_00385 \\
\hline 19 & group_15040 & Transglycosylase SLT domain-containing protein & 76 & 4.608 & 0.018 & WH-SGI-V-07050_00386 \\
\hline 20 & group_11058 & MgtC/SapB family protein & 84 & 4.203 & 0.018 & WH-SGI-V-07050_00439 \\
\hline \multicolumn{7}{|c|}{ PYSEER } \\
\hline & COG / Gene & Function & Presence & Beta & Irt p & Example \\
\hline 1 & group_8321 & Methyltransferase & 68 & 1.9200 & 0.0001 & WH-SGI-V-07050_00375 \\
\hline 2 & group_15072 & Hypothetical & 80 & 1.6800 & 0.0001 & WH-SGI-V-07050_00461 \\
\hline 3 & group_15041 & Integrating conjugative element protein & 75 & 1.6500 & 0.0002 & WH-SGI-V-07050_00387 \\
\hline 4 & group_11114 & Hypothetical & 140 & 1.5000 & 0.0002 & WH-SGI-V-07050_02801 \\
\hline 5 & group_1519 & SAM-dependent methyltransferase & 79 & 1.5700 & 0.0003 & WH-SGI-V-07050_00374 \\
\hline 6 & group_3009 & 4-alpha-glucanotransferase & 72 & 1.3700 & 0.0003 & WH-SGI-V-07051_05729 \\
\hline 7 & $\mathrm{cls} A$ & Major cardiolipin synthase ClsA & 42 & 1.6900 & 0.0003 & WH-SGI-V-07066_05171 \\
\hline 8 & ydfG & NADP-dependent 3-hydroxy acid dehydrogenase & 73 & 1.6200 & 0.0003 & WH-SGI-V-07050_00440 \\
\hline 9 & group_15062 & TetR/AcrR family transcriptional regulator & 73 & 1.6200 & 0.0003 & WH-SGI-V-07050_00441 \\
\hline 10 & $\begin{array}{l}\text { group_11043 } \\
\text { (arsC_1) }\end{array}$ & Arsenate reductase (glutaredoxin) & 78 & 1.5500 & 0.0003 & WH-SGI-V-07050_00379 \\
\hline 11 & acr3 & ACR3 family arsenite efflux transporter & 78 & 1.5500 & 0.0003 & WH-SGI-V-07050_00380 \\
\hline 12 & arsC_2 & Arsenate reductase & 78 & 1.5500 & 0.0003 & WH-SGI-V-07050_00381 \\
\hline 13 & group_11046 & ArsR family transcriptional regulator & 78 & 1.5500 & 0.0003 & WH-SGI-V-07050_00382 \\
\hline 14 & arsH_1 & Arsenical resistance protein & 78 & 1.5500 & 0.0003 & WH-SGI-V-07050_00378 \\
\hline 15 & flic & Flagellin & 109 & 1.2400 & 0.0004 & WH-SGI-V-07064_05796 \\
\hline 16 & group_5435 & Alpha/beta fold hydrolase & 101 & -1.1300 & 0.0004 & WH-SGI-V-07049_05847 \\
\hline 17 & group_5843 & Hypothetical & 35 & 1.8200 & 0.0004 & WH-SGI-V-07066_05167 \\
\hline 18 & group_2314 & Hypothetical & 71 & 1.5400 & 0.0004 & WH-SGI-V-07050_00373 \\
\hline 19 & group_832 & Hypothetical & 77 & 1.5100 & 0.0004 & WH-SGI-V-07050_00384 \\
\hline 20 & group_11053 & Integrating conjugative element relaxase, PFGI-1 class & 77 & 1.5000 & 0.0005 & WH-SGI-V-07050_00417 \\
\hline
\end{tabular}

Presence $=$ number of isolates (of 280) gene is present in. OR = Odds ratio and Ben $\mathrm{p}=$ Benjamini-Hochberg corrected $p$ value for Scoary Beta $=$ slope of regression and Irt $\mathrm{p}=$ likelihood ratio test $p$ value using Pyseer. Genes in bold are present in both lists 


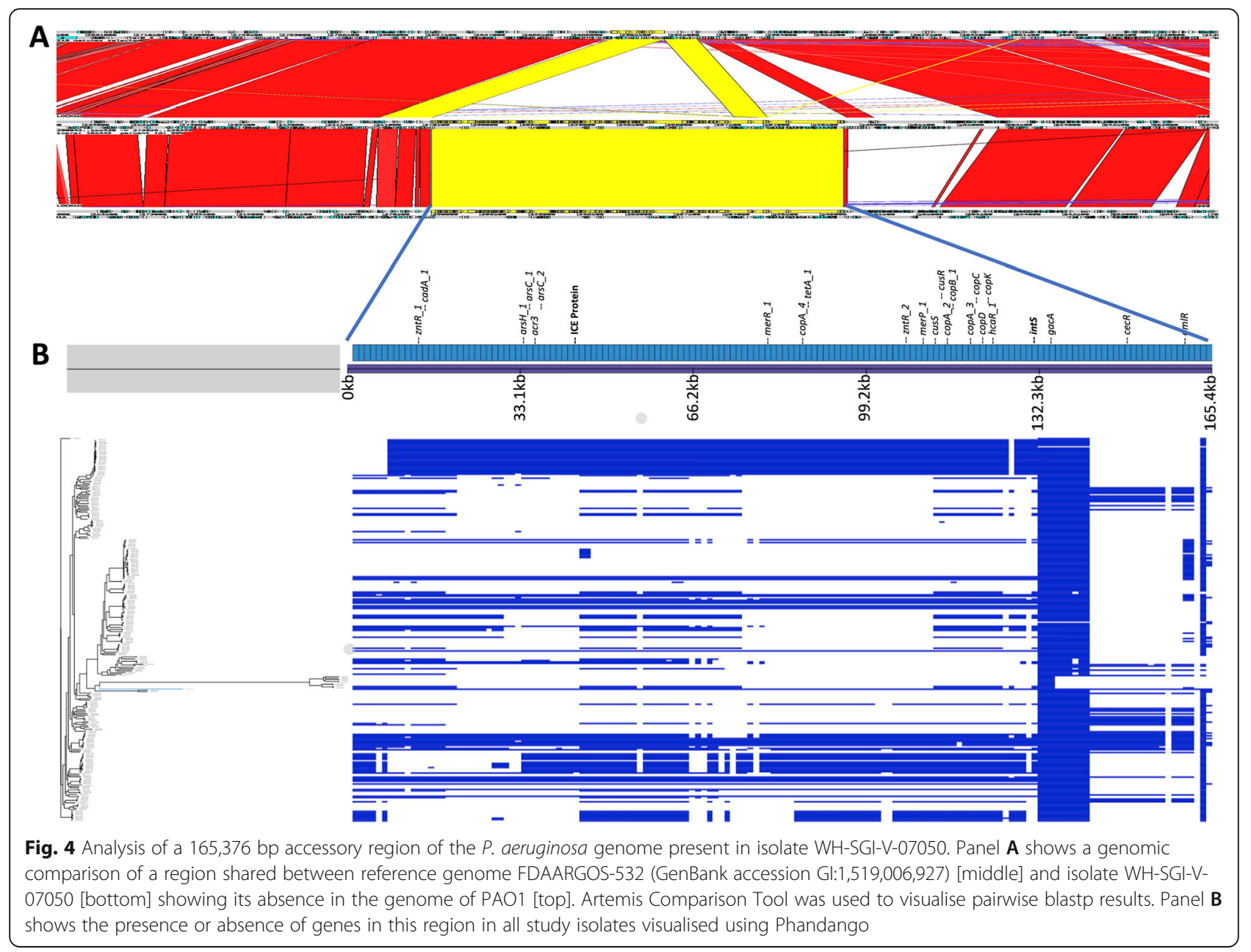

in Table 1 (and raw data in Suppl. Tables 3 and 4). The region also contains a gene for tetracycline resistance (tetA_1) that is significantly associated with this phenotype as well as several transcriptional regulators: - zntR 1, zntR_2, cusR (adjacent to sensor kinase gene cusS), $h c a R_{-} 1, \operatorname{cec} R$ and $d m l R_{-} 24$. Also present in this region are a cluster of nine genes that are present in between 260 and 278 of the 280 genomes studied. These genes include the response regulator gene gacA, a key gene regulating transition from a planktonic to biofilm lifestyle [38], a putative transcriptional regulator gene as well as a colicin receptor gene. The architecture of this region suggests a history of recent recombination events and this is supported by the presence in this region of an Integrative and Conjugative Element (ICE) protein and a prophage integrase that would strongly suggest that these gene clusters have been mobilised by conjugative transposition and phage transduction events into independent lineages.

Transposon mutagenesis can be used to help elucidate the relative importance of individual candidate genes to observed biofilm phenotypes. The ars $R$ family transcriptional regulator (Table 1) is present in 78 of 280 isolates and is not present in PAO1. It is distinct from the ars $R$ gene present in $275 / 280$ of our isolates that forms an arsenic resistance operon with arsB and ars $C$ in PAO1. We found that two separate transposon mutants of the arsR gene in PAO1 showed enhanced biofilm growth at $22{ }^{\circ} \mathrm{C}$ compared to $37{ }^{\circ} \mathrm{C}$ (Suppl. Figure 2). This is supportive of a role for this gene in biofilm production at ambient temperature although several future mutagenesis experiments would be required to support a key role for such genes in this temperature associated phenotype.

\section{Temperature associated core genome SNPs}

Pyseer analysis yielded 2081 significant SNPs (lrt $p<$ 0.001 ) associated with increased biofilm production at $22{ }^{\circ} \mathrm{C}$ compared to $37{ }^{\circ} \mathrm{C}$ (Suppl. Table 5). All SNPs ranked by significance (lrt $p$-values on y-axis) are shown in a Manhattan Plot in Fig. 5 with their location in the PAO1 genome. The 30 most significant hits (ordered by lrt-adjusted $p$-values) relative to the PAO1 genome are 


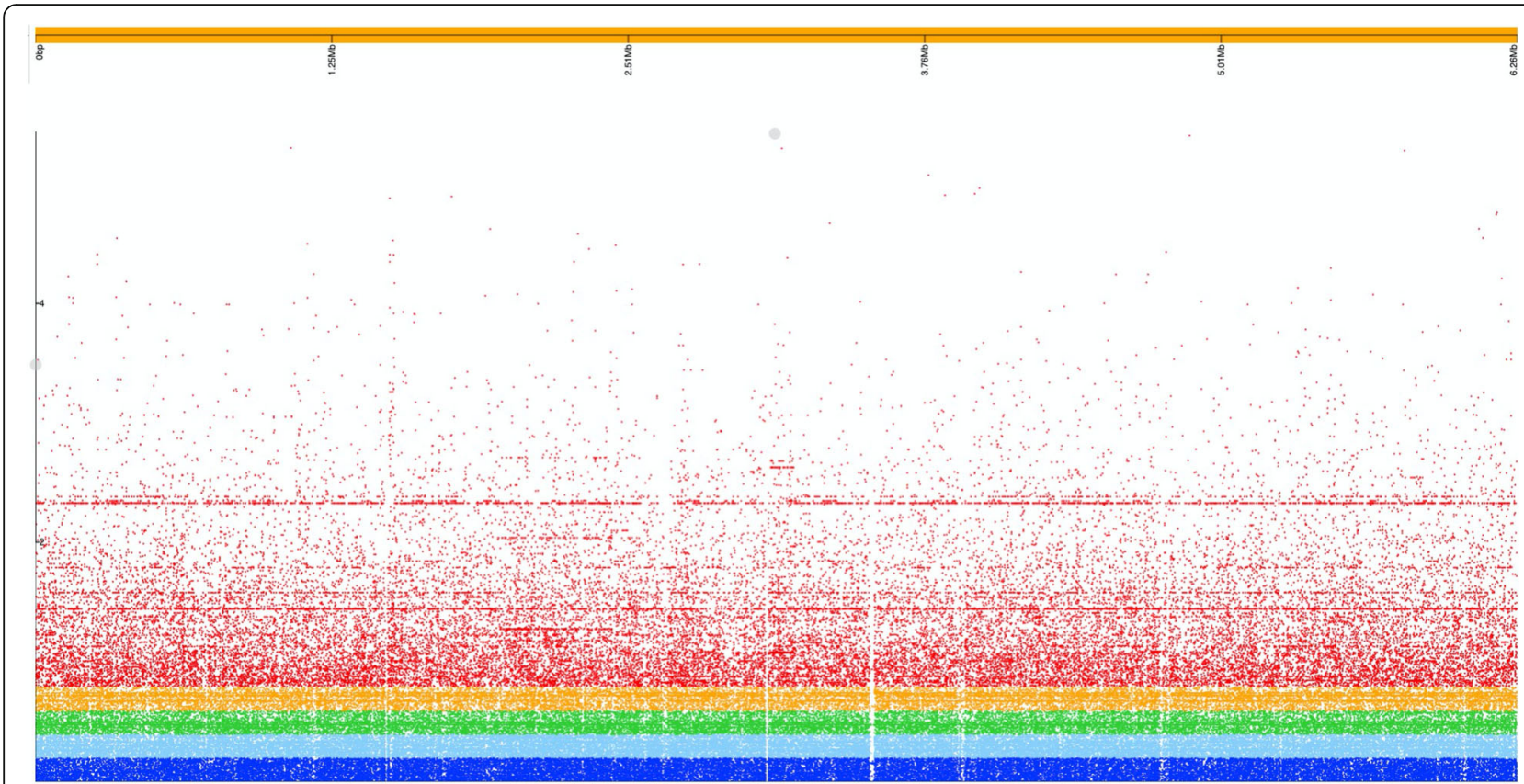

Fig. 5 Manhattan plot showing core genome significance (Irt $p$-value) of SNPs in the PAO1 genome associated with moregreater biofilm production at $22^{\circ} \mathrm{C}$ than at $37{ }^{\circ} \mathrm{C}$. The Y-axis corresponds to Irt $p$ - values. $37^{\circ} \mathrm{C}$ and their position in the PAO1 chromosome

shown in Suppl. Table 6. These include three genes with more than one SNP - Type II secretion system protein $\mathrm{D}$, a sulfotransferase family protein and a nucleoside binding protein. Type II secretion systems are associated with pathogenesis and environmental survival in a number of species and involve the export of proteins to the extracellular biofilm matrix [39]. The possible role of sulfotransferases and the second most significant SNP in $c y s W$, a sulfate transport system permease gene, in biofilm production are unclear as is the role of PAO1 gene 00240, a nucleoside binding protein. The most significant SNP associated with increased biofilm production at $22{ }^{\circ} \mathrm{C}$ is in the PAO1 gene 04493 , that codes for 1-acyl-sn-glycerol-3-phosphate acyltransferase. This enzyme and quiP, an acyl-homoserine lactone acylase gene, may be involved in interactions with QS systems as the major QS signalling molecules in P. aeruginosa are acylhomoserine lactones. Other SNPs in genes that have previously been found to be involved in biofilm production or regulation are in $\operatorname{alg} X$ and $\operatorname{alg} A$, genes that form part of the operon involved in the production of the well-characterized extracellular polysaccharide biofilm component alginate [40] that is associated with the hyper-mucosity phenotype observed in isolates from some cystic fibrosis patients. The response regulator pleD is known to play a role in motility and transition between sessile and motile forms in Caulobacter crescentus[41] and its homologue in PAO1, $d g c H[42]$ is required for biofilm production. Less clear is the role of the helix-turn-helix transcriptional regulator syrM.
Other significant SNPs in genes involved in cellular motility in Table 2 are the flagellar glycosyl transferase gene $f g t A$ and a putative major fimbrial subunit gene lpfA that may facilitate bacterial attachment and promote biofilm formation [43]. A full list of SNPs associated with greater biofilm production at $22{ }^{\circ} \mathrm{C}$ than at $37{ }^{\circ} \mathrm{C}$ is shown in Suppl. Table 6.

\section{Biofilms on stainless-steel}

Of the isolates assessed for biofilm production on stainless-steel (Suppl. Figures 1), $40 \%$ ( $n=10$ out of 25 ) ST111 and $52 \%$ ( $n=13$ out of 23) ST235 were considered high density biofilm producers. This demonstrates a large intra-clone variation of the biofilm phenotype. However, the variation observed in one clone was similar to the other (i.e. not significantly different), suggesting the intra-clone variation may be a common feature of other clones.

BEAST 2 analysis suggests that this sample of 153 ST111 isolates diverged from a common ancestor $\cong 43$ years ago (Fig. 6.a), with a pan-genome of 15,488 genes. The 107 ST235 isolates included in this study had a pan-genome of 15,178 genes and diverged from a common ancestor $\cong 28$ years ago (Fig. 6.b), suggesting that the two lineages emerged within approximately 15 years of each other. When considered alongside biofilm phenotype, those isolates sharing phylogenetic similarity display similar biofilm phenotype, except for ST235 isolates WH-SGI-V-07622 and 
Table 2 Details of genes / COGs associated with biofilm phenoptype on stainless-steel identified by Pyseer

\begin{tabular}{ll}
\hline ST111 & \\
Cog / Gene & Function \\
group_257 & Type IV secretion system apparatus / Translocase \\
cusS_2 & Sensor kinase CusS \\
group_2942 & HD-GYP domain-containing protein \\
group_2943 & Hypothetical \\
group_2944 & Hypothetical \\
group_2945 & Transposase \\
group_2946 & XRE family transcriptional regulator \\
group_2947 & type II toxin-antitoxin system RelE/ParE family toxin \\
hin_4 & DNA-invertase \\
group_2949 & Tn3 family transposase
\end{tabular}

$\begin{array}{llll} & \text { Presence } & \text { Irt-pvalue } & \text { Beta } \\ \text { WH-SGI-V-07174_06314 } & 16 & 0.005 & -0.773 \\ \text { WH-SGI-V-07168_02759 } & 22 & 0.008 & 1.050 \\ \text { WH-SGI-V-07174_02964 } & 2 & 0.008 & -1.820 \\ \text { WH-SGI-V-07174_02965 } & 2 & 0.008 & -1.820 \\ \text { WH-SGI-V-07174_02966 } & 2 & 0.008 & -1.820 \\ \text { WH-SGI-V-07174_02967 } & 2 & 0.008 & -1.820 \\ \text { WH-SGI-V-07174_02968 } & 2 & 0.008 & -1.820 \\ \text { WH-SGI-V-07174_02969 } & 2 & 0.008 & -1.820 \\ \text { WH-SGI-V-07174_02970 } & 2 & 0.008 & -1.820 \\ \text { WH-SGI-V-07174_02971 } & 2 & 0.008 & -1.820\end{array}$

\section{ST235}

\section{Cog / Gene Function}

group_2587 Type IV secretion system apparatus / Translocase

group_2588 Hypothetical

group_2593 HK97 gp10 family phage protein

group_977 Methyltransferase domain protein

group_129 Glycoside hydrolase family 19 protein

group_2655 Hypothetical

merP_2 Mercuric transport protein periplasmic component

group_953 Mercury resistance co-regulator MerD

group_596 Phage gp6-like head-tail connector protein

$\mathrm{dcm} \quad$ DNA-cytosine methyltransferase

algA_3 Alginate biosynthesis protein AlgA

group_199 SAM-dependent methyltransferase, partial

group_2651 Hypothetical

group_2634 Hypothetical

group_2635 Hypothetical

group_2636 DUF3577 domain-containing protein

group_2638 Hypothetical

group_2639 TIGR03761 family integrating conjugative element protein

frmR

group_2677 Transcriptional repressor FrmR

group_994

Hypothetical

group_1012

Hypothetical

group_1013

Uridylate kinase

group_1014

DUF3275 family protein

group_1015

SAM-dependent methyltransferase

group_1016

Hypothetical

group_1017

TIGR03759 family integrating conjugative element protein

group_1018

Type IV conjugative transfer system coupling protein TraD

group_1019

TIGR03747 family integrating conjugative element membrane protein

WH-SGI-V-07406_05831

Presence

Irt-pvalue

Beta

WH-SGI-V-07406_05832

0.000

1.640

WH-SGI-V-07406_05844

0.000

1.640

WH-SGI-V-07406_05845

0.000

1.640

WH-SGI-V-07425_03679

0.000

1.640

WH-SGI-V-07425_03618

0.001

1.920

WH-SGI-V-07176_04878

0.001

1.920

WH-SGI-V-07176_04876

0.001

$-2.240$

WH-SGI-V-07406_05848

0.001

$-2.240$

WH-SGI-V-07170_03311

0.001

1.560

WH-SGI-V-07170_03301

0.002

2.600

WH-SGI-V-07176_05804

0.002

2.600

WH-SGI-V-07425_03614

0.003

2.420

WH-SGI-V-07425_01793 4

0.005

1.510

WH-SGI-V-07425_01794

0.006

1.850

WH-SGI-V-07425_01796

0.006

1.850

WH-SGI-V-07425_01801

0.006

1.850

WH-SGI-V-07425_01809

0.006

1.850

WH-SGI-V-07425_04778

0.006

1.850

WH-SGI-V-07425_04812

0.006

1.850

WH-SGI-V-07425_01799

0.006

1.850

0.006

1.850

WH-SGI-V-07496_01971

0.007

$-3.470$

WH-SGI-V-07496_01976

0.007

$-3.470$

WH-SGI-V-07496_01979 3

0.007

$-3.470$

WH-SGI-V-07496_01983

0.007

$-3.470$

WH-SGI-V-07496_01984 3

0.007

$-3.470$

WH-SGI-V-07496_01987

0.007

$-3.470$

WH-SGI-V-07496_01988

0.007

$-3.470$

WH-SGI-V-07496_01991

0.007

$-3.470$

group_1020

GIY-YIG nuclease family protein

0.007

$-3.470$ 
Table 2 Details of genes / COGs associated with biofilm phenoptype on stainless-steel identified by Pyseer (Continued)

\begin{tabular}{|c|c|c|c|c|c|}
\hline group_1021 & TIGR03745 family integrating conjugative element membrane protein & WH-SGI-V-07496_01998 & 3 & 0.007 & -3.470 \\
\hline group_1022 & TIGR03746 family integrating conjugative element protein & WH-SGI-V-07496_02000 & 3 & 0.007 & -3.470 \\
\hline group_1023 & TIGR03752 family integrating conjugative element protein & WH-SGI-V-07496_02002 & 3 & 0.007 & -3.470 \\
\hline group_1024 & Conjugative transfer ATPase & WH-SGI-V-07496_02004 & 3 & 0.007 & -3.470 \\
\hline group_1025 & Tral domain-containing protein & WH-SGI-V-07496_02019 & 3 & 0.007 & -3.470 \\
\hline $\mathrm{ftsH} 4$ & ATP-dependent zinc metalloprotease Fts $\mathrm{H} 4$ & WH-SGI-V-07496_03490 & 3 & 0.007 & -3.470 \\
\hline
\end{tabular}

Presence $=$ number of genomes gene is present in /25 for ST111 and /23 for ST235

WH-SGI-V-07625, as well as isolates WH-SGI-V07498 and WH-SGI-V-07624. Nevertheless, this analysis suggests many higher and lower density biofilm formers are closely related, and that biofilm production on stainless-steel is not necessarily predictable based only on genomic analysis.

The pan-genome of biofilm genes widely described in the literature (Fig. 7) has a different core vs. accessory structure in each of the two lineages in this study, with the majority of genes (approximately $54 \%$ ) in both lineages, present in fewer than $15 \%$ of genomes. The ST235 isolates analysed here have a higher percentage of core genes compared to ST111 (61.7\% vs. $31.34 \%)$, whilst ST111 has a larger cloud genome compared to ST235 (40.3\% vs. $17.02 \%)$. This variation is due to a greater number of homologues of genes in the ST111 biofilm pan-genome pelA has four homologues in ST111 isolates and flgK, pilY1, pslI and pslJ have two each. The genes pilA and fimT were both identified in the ST111 pan-genome, but not found in the ST235 pan-genome, whilst pslC was found in ST235 but not in the ST111 pan-genome.

Pan-genome association analysis of stainless-steel biofilm phenotype using Scoary on ST111 $(n=25)$ and ST235 $(n=23)$ isolates showed no significant associations after Bonferroni or Benjamini-Hocht corrections for multiple sampling $(p<0.05)$. However, Pyseer allows pangenome association analysis using mean $\mathrm{OD}_{540}$ values and the most statistically significant associations between accessory gene presence and biofilm production on stainless-steel are shown in Table 3. In ST111 and ST235 the most significantly associated genes are small

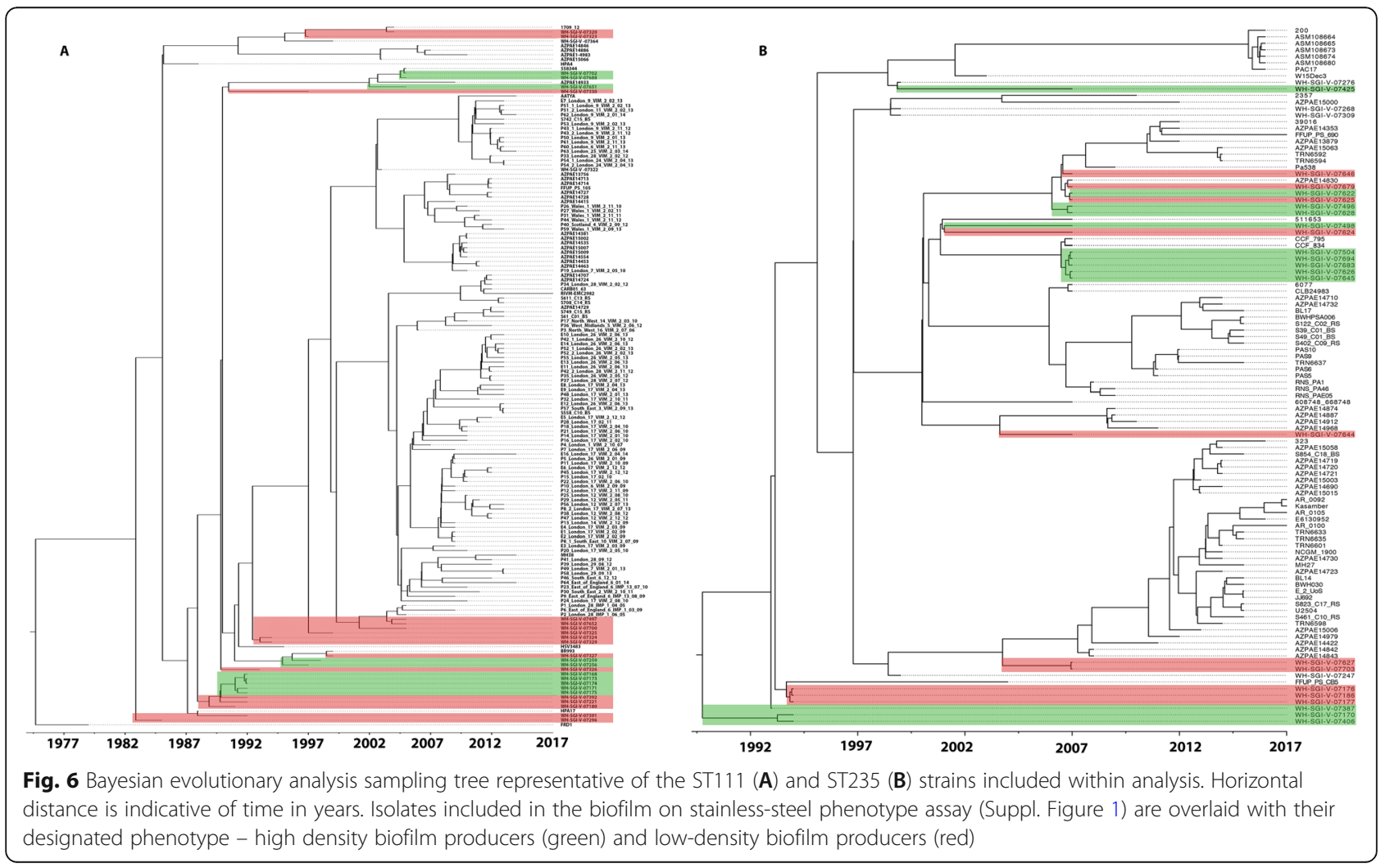




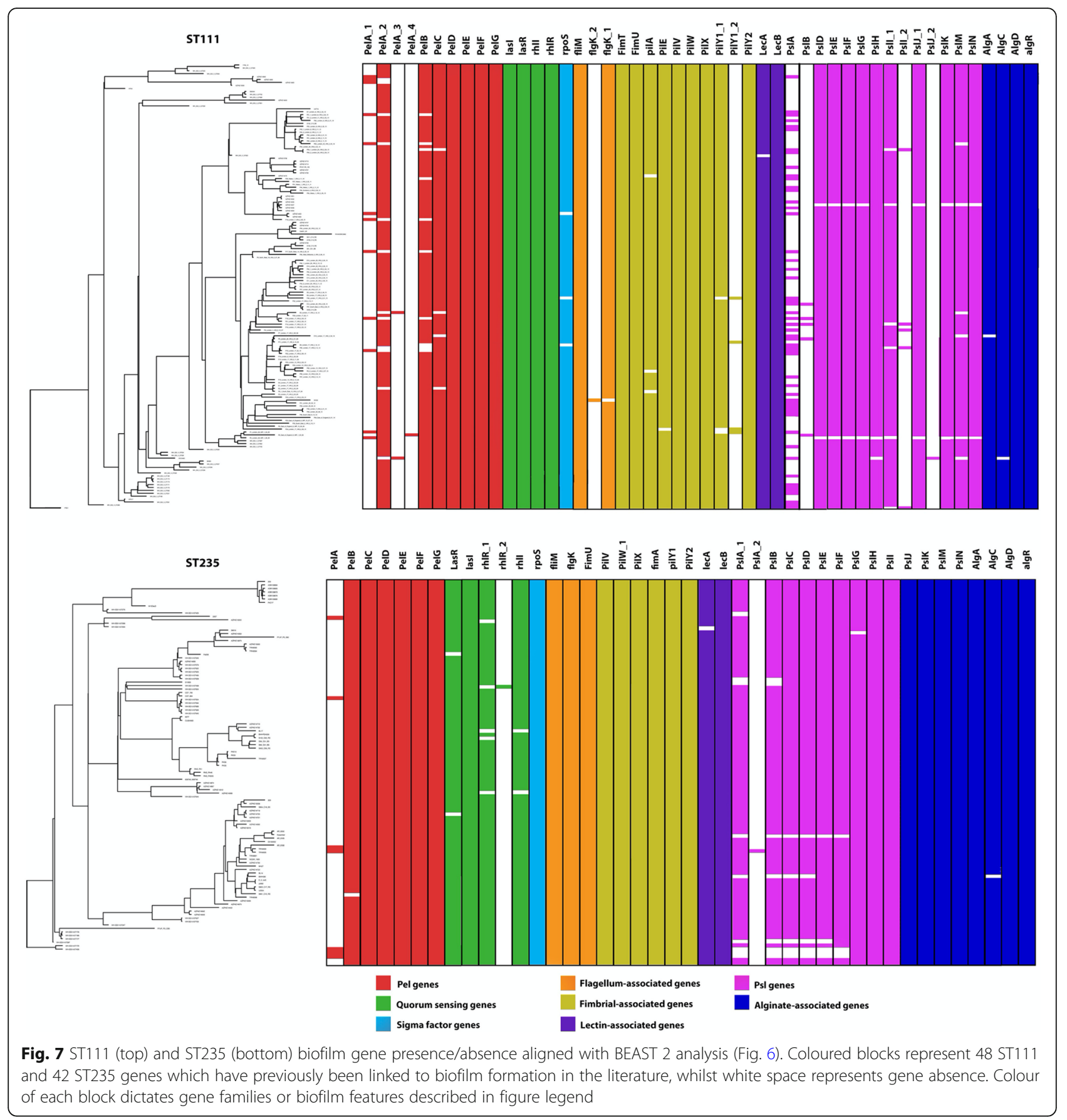

proteins of 84 and 86 amino-acids respectively that share $21 \%$ amino acid sequence identity to each other and only match to hypothetical proteins using blastp. HHpred indicate partial but significant structural similarity to the same $H$. pylori Type IV secretion system translocase for both proteins. Protein translocation by these previously uncharacterised putative translocases could have a significant role in biofilm production through extracellular transport contributing to biofilm mass. In ST111 isolates other genes with significant associations include the sensor kinase cusS (also associated with temperature dependent biofilm production above) found in 22 isolates, and eight genes located together and present in only two ST111 isolates (isolate WH-SGI-V-07174 genes 02964-02971) that include two transposases, a type II toxin/antitoxin (TA) system RelE/ ParE family toxin, and an XRE family transcriptional regulator that possibly represents an integrated plasmid sequence. Secreted TA system toxins have previously been found to be involved in biofilm formation in $P$. aeruginosa 
Table 3 Details of genes / COGs associated with biofilm phenoptype on stainless-steel identified by Pyseer

\begin{tabular}{|c|c|c|c|c|c|}
\hline \multicolumn{6}{|l|}{ ST111 } \\
\hline Cog / Gene & Function & & Presence & Irt-pvalue & Beta \\
\hline group_257 & Putative Type IV secretion system translocase & WH-SGI-V-07174_06314 & 16 & 0.005 & -0.773 \\
\hline cusS_2 & Sensor kinase CusS & WH-SGI-V-07168_02759 & 22 & 0.008 & 1.050 \\
\hline group_2942 & HD-GYP domain-containing protein & WH-SGI-V-07174_02964 & 2 & 0.008 & -1.820 \\
\hline group_2943 & Hypothetical & WH-SGI-V-07174_02965 & 2 & 0.008 & -1.820 \\
\hline group_2944 & Hypothetical & WH-SGI-V-07174_02966 & 2 & 0.008 & -1.820 \\
\hline group_2945 & Transposase & WH-SGI-V-07174_02967 & 2 & 0.008 & -1.820 \\
\hline group_2946 & XRE family transcriptional regulator & WH-SGI-V-07174_02968 & 2 & 0.008 & -1.820 \\
\hline group_2947 & type II toxin-antitoxin system RelE/ParE family toxin & WH-SGI-V-07174_02969 & 2 & 0.008 & -1.820 \\
\hline hin_4 & DNA-invertase & WH-SGI-V-07174_02970 & 2 & 0.008 & -1.820 \\
\hline group_2949 & Tn3 family transposase & WH-SGI-V-07174_02971 & 2 & 0.008 & -1.820 \\
\hline \multicolumn{6}{|l|}{ ST235 } \\
\hline Cog / Gene & Function & & Presence & Irt-pvalue & Beta \\
\hline group_2587 & Putative Type IV secretion system translocase & WH-SGI-V-07406_05831 & 5 & 0.000 & 1.640 \\
\hline group_2588 & Hypothetical & WH-SGI-V-07406_05832 & 5 & 0.000 & 1.640 \\
\hline group_2593 & HK97 gp10 family phage protein & WH-SGI-V-07406_05844 & 5 & 0.000 & 1.640 \\
\hline group_977 & Methyltransferase domain protein & WH-SGI-V-07406_05845 & 5 & 0.000 & 1.640 \\
\hline group_129 & Glycoside hydrolase family 19 protein & WH-SGI-V-07425_03679 & 4 & 0.001 & 1.920 \\
\hline group_2655 & Hypothetical & WH-SGI-V-07425_03618 & 4 & 0.001 & 1.920 \\
\hline merP_2 & Mercuric transport protein periplasmic component & WH-SGI-V-07176_04878 & 6 & 0.001 & -2.240 \\
\hline group_953 & Mercury resistance co-regulator MerD & WH-SGI-V-07176_04876 & 6 & 0.001 & -2.240 \\
\hline group_596 & Phage gp6-like head-tail connector protein & WH-SGI-V-07406_05848 & 12 & 0.001 & 1.560 \\
\hline $\mathrm{dcm}$ & DNA-cytosine methyltransferase & WH-SGI-V-07170_03311 & 19 & 0.002 & 2.600 \\
\hline algA_3 & Alginate biosynthesis protein AlgA & WH-SGI-V-07170_03301 & 19 & 0.002 & 2.600 \\
\hline group_199 & SAM-dependent methyltransferase, partial & WH-SGI-V-07176_05804 & 4 & 0.003 & 2.420 \\
\hline
\end{tabular}

Presence $=$ number of genomes gene is present in $/ 25$ for ST111 and /23 for ST235

as well playing important roles in pathogenicity and persistence [44]. In ST235 genes associated with enhanced biofilm production on stainless-steel include two genes involved in mercury resistance, two methyltransferase proteins and an alginate biosynthesis gene algA_3. The putative ST235 T4SS translocase (WH-SGI-V-07406 gene 05831) is present in five ST235 isolates as are three genes in close proximity (05832, 05844 and 05845 in this isolate) that may represent part of a mobile genetic structure.

Core genome SNPs associated with biofilm production on stainless-steel were found using Pyseer and the most significant hits are shown in Table 4. In ST111 these include a SNP in a GGDEF and EAL domain-containing protein. Such proteins are known to have a key role in cell signalling and biofilm production [45]. The chemotaxis protein McpU and the PAS domain-containing protein are involved in cell-signalling and play a role in biofilm formation. Polymorphisms in the membrane proteins MprF and the DedA membrane family protein may have an effect on biofilm formation through increased translocation of proteins and other macromolecules from the cell. In ST235 the most significant core genome SNP is in a tRNA-Asn region however its relevance to biofilm production on stainless-steel is unclear. Four significant SNP sites are present in the topoisomerase primase domain-containing protein DnaG although the possible role of primase genes in biofilm production or regulation is obscure. Similarly, the importance of SNPs associated with biofilm phenotype for GTPase, ViaA and the two glycoside hydrolase family 19 proteins is unclear although we theorise that such glycoside hydrolases could be involved in biofilm matrix hydrolysis promoting transition of $P$. aeruginosa from a sessile to a planktonic state.

\section{Discussion}

In this study we demonstrate the ability of recently developed bacterial genome and pangenome analysis tools to discover accessory genes and core-genome SNPs that are associated with two different biofilm phenotypes. 
Table 4 Details of SNPs with significant associations with biofilm phenotype on stainless-steel within ST111 and ST235 genomes. Genes containing > 1 SNP site are in bold

\begin{tabular}{|c|c|c|c|}
\hline \multicolumn{4}{|l|}{ ST111 } \\
\hline variant & Gene product & Irt-pvalue & beta \\
\hline 1420097_C_T & Transglycosylase SLT domain-containing protein & 7.790E-03 & $1.160 \mathrm{E}+00$ \\
\hline 5765510_C_T & GGDEF and EAL domain-containing protein & $7.790 \mathrm{E}-03$ & $1.160 \mathrm{E}+00$ \\
\hline 3148782_G_A & hypothetical protein & 7.790E-03 & $1.160 E+00$ \\
\hline 5733260_G_A & Putative 2-dehydropantoate 2-reductase & $7.790 \mathrm{E}-03$ & $1.160 \mathrm{E}+00$ \\
\hline 3227055_G_T & DedA membrane protein family & 7.790E-03 & $1.160 \mathrm{E}+00$ \\
\hline 460063_G_T & EcsC family protein & 7.790E-03 & $1.160 \mathrm{E}+00$ \\
\hline 1590045_A_G & Methyl-accepting chemotaxis protein McpU & 7.790E-03 & $1.160 E+00$ \\
\hline 788564_G_A & Integral membrane protein mprF [Brucella suis] & $7.790 \mathrm{E}-03$ & $1.160 \mathrm{E}+00$ \\
\hline 124758C_G & PAS domain-containing protein & $9.980 \mathrm{E}-03$ & 9.950E-01 \\
\hline \multicolumn{4}{|l|}{ ST235 } \\
\hline variant & Gene & Irt-pvalue & beta \\
\hline 3107052_C_T & tRNA-Asn(gtt) & $1.14 \mathrm{E}-05$ & $1.95 \mathrm{E}+00$ \\
\hline 1737948_T_C & DUF1983 domain-containing protein & 1.08E-04 & $2.18 \mathrm{E}+00$ \\
\hline 1580887_A_G & GTPase & $3.45 \mathrm{E}-04$ & $2.33 \mathrm{E}+00$ \\
\hline 1549594_C_T & Relaxase & 3.45E-04 & $2.33 \mathrm{E}+00$ \\
\hline 1580485_A_G & GTPase & $3.45 \mathrm{E}-04$ & $2.33 \mathrm{E}+00$ \\
\hline 3128027_C_G & Glycoside hydrolase family 19 protein & $3.52 \mathrm{E}-04$ & $2.15 \mathrm{E}+00$ \\
\hline 4192259_C_G & Glycoside hydrolase family 19 protein (n.b. different gene to above) & 3.52E-04 & $2.15 E+00$ \\
\hline 1553088_A_G & Conjugal transfer protein TraG N-terminal domain-containing protein & 4.58E-04 & $-2.91 E+00$ \\
\hline 1565427_G_C & TIGR03758 family integrating conjugative element protein & $4.58 \mathrm{E}-04$ & $-2.91 E+00$ \\
\hline 3128516_G_A & Hypothetical protein & $5.38 \mathrm{E}-04$ & $1.68 \mathrm{E}+00$ \\
\hline 3128498_C_G & Hypothetical protein & $5.38 \mathrm{E}-04$ & $1.68 \mathrm{E}+00$ \\
\hline 312896T_G & VWA domain protein viaA & $5.38 \mathrm{E}-04$ & $1.68 \mathrm{E}+00$ \\
\hline 1584342_T_C & DNA topoisomerase III & 6.85E-04 & $1.82 \mathrm{E}+00$ \\
\hline 310354C_T & Type I restriction endonuclease subunit $R$ & 1.17E-03 & $2.64 \mathrm{E}+00$ \\
\hline 3101715_A_T & topoisomerase primase domain-containing protein dnaG & 1.17E-03 & $2.64 \mathrm{E}+00$ \\
\hline 3101859_T_C & topoisomerase primase domain-containing protein dnaG & 1.17E-03 & $2.64 \mathrm{E}+00$ \\
\hline 3101802_G_A & topoisomerase primase domain-containing protein dnaG & 1.17E-03 & $2.64 \mathrm{E}+00$ \\
\hline 3102546_C_T & topoisomerase primase domain-containing protein dnaG & 1.17E-03 & $2.64 \mathrm{E}+00$ \\
\hline 312840T_C & VWA domain protein viaA & $2.21 \mathrm{E}-03$ & $1.55 \mathrm{E}+00$ \\
\hline 309466T_G & Type I restriction endonuclease subunit $R$ & $2.92 \mathrm{E}-03$ & $1.74 \mathrm{E}+00$ \\
\hline
\end{tabular}

This resulted in our discovery of a genetic region containing many heavy metal resistance genes (arsenic, copper, mercury and cadmium) that we found associated with increased biofilm production at $22{ }^{\circ} \mathrm{C}$ compared to $37^{\circ} \mathrm{C}$ and core genome SNPs in biofilm associated genes such as $\operatorname{alg} A$, $\operatorname{alg} X$, type II secretion system protein $\mathrm{D}$ gene $x p s D$ and response regulator genes such as pleD and quiP that are also significantly associated with this phenotype and have previously been shown to have roles in biofilm biosynthesis and quorum sensing. Pangenome associations with biofilm production by isolates of
ST111 and ST235 on stainless-steel similarly revealed novel accessory genes including ones with homology to a T4SS translocase and a type II TA system toxin protein as well as an alginate accessory gene algA_3 and two mercury resistance genes. We also identified several SNPs in cell-signalling and cell-membrane protein genes that are associated with enhanced biofilm production on stainless-steel. It is not clear why possession of genetic regions coding for heavy metal resistance should results in enhanced biofilm production at lower temperatures but we hypothesize that such regions may be associated 
with overall genotypes that have adapted for survival in such environments.

Whilst the phenotype and genotype analysis and associations described in this paper provide novel insight into the relationship between non-discreet phenotypes and genotype, standardization of such phenotypes are required. This will be essential if GWAS and pan-GWAS studies are to be utilized to better understand the genetics of biofilm production. Central to this is the choice of methodologies for association analysis. In our pangenome analysis we found Scoary to be useful in identifying accessory genes associated with biofilm phenotype in our larger sample experiments (temperature-dependent biofilm) but lacked power in studying smaller groups of isolates. Pyseer proved more useful in analysing smaller datasets (stainless-steel biofilm analysis) in this study possibly due to the inclusion of continuous phenotype data rather than binary phenotypes in association studies that is not possible using Scoary. When both Scoary and Pyseer were used to analyse the same large dataset (280 isolates) they both identified many of the same accessory genes (Table 1) although for Scoary we found the use of Bonferroni (FWER) corrected $p$-values was too restrictive and Benjamini-Hochberg (FDR) corrected $p$-values may be more appropriate to such analyses.

\section{Conclusions}

We were able to identify candidate genes in which polymorphism (or deletion) was associated with differences in temperature-dependent biofilm phenotype for 280 P. aeruginosa isolates. The two different GWAS approaches used in this paper (SNP-based and gene presence / absence) both resulted in the identification of some similar genes, The relationships between biofilm phenotypes on stainless-steel and genotype in $P$. aeruginosa was less clear, likely due to the smaller number of isolates used in this study. Further work should seek to utilize GWAS and pan-GWAS studies to identify new gene targets / cellular machinery alongside functional studies such as gene expression (for example RNA-seq). Our study demonstrates the importance of understanding the complex nature of $P$. aeruginosa biofilm, and that phenotypic studies in isolation and/or assumptions that biofilm phenotype is consistent across $P$. aeruginosa offer little in the way of meaningful data. Unravelling the complexity of phenotype-genotype relationships of $P$. aeruginosa will be an essential step in understanding and targeting $P$. aeruginosa biofilms as part of possible therapeutic strategies to tackle the threat posed by this major MDR pathogen.

\section{Methods}

\section{Bacterial isolates and genome sequences}

Isolates in this study were collected and genome sequenced in a previous study by van Belkum et al., 2015
[6]. They comprise isolates from the bioMérieux private clinical strain collection (218 isolates) and isolates from a study by Pirnay et al., 2009 [10] (62 isolates). This study analysed two different sets of these $P$. aeruginosa isolates. The first set, hereafter described as 'temperature-dependent biofilm analysis' contained all 280 isolates details of which are shown in Suppl. Tables 1, this study and Suppl. Table ST1 in van Belkum et al., 2015 [6].

The second set, hereafter described as 'stainless-steel biofilm analysis' contained 260 P. aeruginosa genomes (Suppl. Table 2), 153 of which belong to ST111 and 107 to ST235. Of these, 25 ST111 and 23 ST235 isolates were used to study biofilm phenotypes in vitro. All genomic data were downloaded from Genbank as Fasta nucleotide sequences. Bacterial isolates used for biofilm phenotyping were provided by bioMérieux (France) and Synthetic Genomics (USA) and were analysed in two previously published studies [6,7].

\section{Construction of phylogenies}

Core genome Single Nucleotide Polymorphisms (SNPs) were identified using the kSNP3 (v3.1) pipeline [26]. Kmer lengths of 21 were used for temperature-dependent biofilm analysis, whilst for stainless-steel biofilm analysis, kmer lengths for ST111 and ST235 were 21 and 23 nucleotides respectively. Kmer lengths were selected using the KCHOOSER module within the kSNP3 suite which provides the optimum kmer length for each dataset. Bayesian phylogenetic analysis was performed using BEAST 2 (v2.4.7) [46]. The following conditions were set based on those used in a phylogenetic study of the $P$. aeruginosa MDR ST111 lineage [9] - gamma site heterogeneity model, Hasegawa-Kishino-Yano (HKY) substitution model, relaxed-clock log-normal, chain length 5,000,000. BEAST 2 output was summarized using TreeAnnotator with a $5 \%$ burn in.

\section{Construction and interrogation of pan-genome}

All genomes were annotated using PROKKA (v1.12) [47]. Genome Feature Files (GFF3) produced by PROKKA were used as input for Roary (v3.11.2) [48] pan-genome analysis using default settings. In brief Roary; converts input files into protein sequences, filters partial sequences and then performs an allagainst-all comparison with BLASTP with a default percentage sequence identity of $95 \%$. Outputs were visualized with the interactive visualization tool Phandango v1.3.0 [49]. Pangenome terminology is used as described by Roary; for example core genes $(99 \%<=$ strains $<=100 \%)$, cloud genes $(0 \%<=$ strains $<15 \%)$. Non-core genes are considered accessory genes whilst 
the pan-genome is considered to be all the genes found within a cohort of genomes.

\section{Biofilm phenotypes - Temperature-dependent biofilm analysis}

The method for biofilm growth was a modified version of that described by Coffey and Anderson [50]. $P$. aeruginosa strains were maintained on LuriaBertani (LB) agar (BD, Sparks, MD) at $4{ }^{\circ} \mathrm{C}$. Liquid cultures were prepared by inoculating a single colony into $10 \mathrm{ml}$ of LB broth (BD, Sparks, MD), and incubated overnight at $37{ }^{\circ} \mathrm{C}$ with agitation $(180 \mathrm{rpm})$. This was diluted to an optical density (OD) of $1.0_{540}$ and a 1:100 inoculation in sterile LB broth was made in standard non-modified 96-well polystyrene plates (Starsted, Leicester, UK). Plates were incubated without shaking at either 22 or $37^{\circ} \mathrm{C}$ for $48 \mathrm{~h}$.

\section{Biofilm phenotypes - Stainless-steel biofilm analysis}

Stainless-steel biofilm analysis was performed on MBEC biofilm 96-well plates (Innovotech, Edmonton, Canada), with pegged lids spray coated with stainless-steel. The sub-micron thick stainless-steel coatings were deposited onto the lid by the physical vapour deposition technique of magnetron sputtering, which is widely used in industry to deposit thin (sub-micron to several micron thick) metallic or ceramic coatings onto a wide range of components [51]. The coatings were sputtered from a $300 \mathrm{~mm} \times 100 \mathrm{~mm} 304$ grade stainless-steel target in a UDP350 coating rig (Teer Coatings Ltd).

An overnight culture of each strain was diluted to an optical density (OD) of $1.0_{540}$. A 1:100 inoculation in sterile LB broth was made in a well of the MBEC plate base $(n=4$ per isolate).

The MBEC lid was replaced and the plate was incubated at $37{ }^{\circ} \mathrm{C}$ with agitation (110 rpm) for $48 \mathrm{~h}$, with a change to a new 96-well plate with $200 \mu \mathrm{l}$ of sterile LB broth in each well after $24 \mathrm{~h}$.

\section{Biofilm phenotypes - quantifying}

To quantify biofilms, plates or pegs were gently immersed in water to remove non-adhered cells, placed in $0.1 \%$ crystal violet (w/v) (Sigma, Dorset) for $10 \mathrm{~min}$ then gently washed in water, twice, to remove excess stain. The plates for temperature-dependent biofilm analysis had $200 \mu \mathrm{l}$ of $30 \%$ acetic acid (v/v) added to each well to solubilize stain, whereas the lid with stained pegs was placed into a 96-well plate containing $200 \mu \mathrm{l}$ per well of $30 \%$ acetic acid (Fisher Scientific, Loughborough) for $10 \mathrm{~min}$ with agitation $(110 \mathrm{rpm})$. Optical density of each well was measured at $540 \mathrm{~nm}$ using a FLUOstar Omega plate reader (BMG Labtech, Aylesbury).

\section{Trait definition}

In order to carry out GWAS and pan-GWAS (below), categorization of biofilm production was required. Currently, no agreed method exists for the categorization of higher density or lower density biofilm formers, and the wider biofilm literature uses a dynamic range of methods, characteristics and approaches to such studies [52]. For temperature-dependent biofilm analysis, isolates were split into one of two traits: either producing more biofilm at $37{ }^{\circ} \mathrm{C}$ compared to growth at $22{ }^{\circ} \mathrm{C}$, or producing more biofilm at $22{ }^{\circ} \mathrm{C}$ compared to $37{ }^{\circ} \mathrm{C}$ (Fig. 2 and Suppl. Table 1). As part of our stainless-steel biofilm analyses, a 'higher density' biofilm formation trait was considered to be exhibited in strains providing an OD reading $>=0.5_{540}$, whilst 'lower density' biofilm formation was considered as an OD reading of $<0.5_{540}$. Defining the stainless-steel biofilm trait as such provides statistically significant differences $(p<0.05)$ when comparing the two sets of optical density data. Similar approaches to arbitrarily classifying continuous phenotypes have been used in previous bacterial GWAS studies [53].

\section{Pan-genome-wide association analysis}

We used two GWAS analysis tools to investigate pangenome associations with biofilm phenotypes:- Scoary (v.1.6.9) [54] and Pyseer (v.1.3.3) [55]. These have been developed specifically for the study of bacterial datasets. The input for both Scoary and Pyseer are the gene presence / absence file generated by Roary and a traits file containing isolate name and either a binary or categorical value. Pyseer can also use quantitative (continuous) phenotype data as input. Associations in Scoary are derived from scoring the correlation of each gene in the accessory genome with phenotype using Fisher's exact test before correcting for spurious associations caused by stratification due to phylogenetic structure. The key outputs of Scoary are a list of genes or Common Orthologous Groups (COGs) with an odds ratio and associated $p$-values corrected for erroneous false positives due to multiple testing using both false discovery rate (FDR) [56] and family-wise error rate (FWER) [57] approaches commonly called Benjamini-Hochberg and Bonferroni corrections respectively. Pyseer uses linear regression models adjusted for stratification in population structure and outputs a beta value (slope of the regression line) with associated likelihood ratio test (lrt) $p$ values.

Analyses were performed independently for temperature-dependent biofilm traits as for both ST111 lineage and ST235 lineage genomes in the stainless-steel biofilm analysis. Genes found to have 
statistically significant associations with biofilm phenotype were further annotated using Blastp and if no hit was found, assessed using the protein homology detection, function and structure prediction tool HHpred (MPI Bioinformatics Tool Kit) [58]. The top three HHpred predictions were considered for assumed protein identity and function, assuming a match probability of at least $80 \%$.

\section{SNP genome-wide association study}

In the temperature-dependent biofilm analysis core genome SNPs relative to a PAO1 reference genome [59] were identified using kSNP3 and we then used Pyseer to identify SNPs associated with biofilm phenotype. Core genome SNPs were identified separately in our stainless-steel biofilm analyses by kSNP3 using ST111 strain FRD1 [60] and ST235 strain NCGM2 [61] as references. Analysis was performed independently for temperature-dependent analysis as before for both ST111 lineage and ST235 lineage genomes in the stainless-steel dataset. The stainlesssteel dataset was analysed by Scoary using binary phenotype data $\left(1=\mathrm{OD}_{540}>=0.5\right.$ and $0=\mathrm{OD}_{540}<$ 0.5 ) and by Pyseer using continuous data (mean $\mathrm{OD}_{540}$ values). SNPs significantly associated with phenotype were located in the PAO1 genome using Artemis genome browser [62]. Genes containing SNPs of interest were interrogated using BlastP and HHpred, with the top three predictions with a probability match of over $80 \%$ considered for assumed protein function.

\section{Abbreviations \\ BLAST: Basic local alignment search tool; bp: Base pair(s); COGs: Clusters of orthologous groups; GWAS: Genome-wide association study; ICE: Integrating conjugative element; kb: Kilobase pair(s); Irt: Likelihood-ratio test; MDR: Multidrug resistant; MLST: Multilocus sequence typing; OD: Optical density; QS: Quorum sensing; SAM: S-adenosyl methionine; SNP: Single nucleotide polymorphism; ST: Sequence type; WGS: Whole genome sequencing}

\section{Supplementary Information}

The online version contains supplementary material available at https://doi. org/10.1186/s12864-021-07818-5

Additional file 1: Suppl. Figure S1. Optical density readings at $540 \mathrm{~nm}$ of Pseudomonas aeruginosa biofilm after staining with $0.1 \%$ crystal violet and solubilised in $30 \%$ acetic acid. Biofilms were grown on a modified MBEC assay plate, of which the pegs had been coated in stainless-steel. Data represent twenty-five ST111 (A) and twenty-three ST235 (B) strains. An optical density cut off of $0.5_{540}$ was used to differentiate the statistically significant groups of high density (green) and low density (red) biofilm producers. Significance $(P<0.05)$, as assessed by T-Test is denoted by *. Error bars represent standard deviation. $n=4$ for each isolate. Suppl. Figure S2. Optical density related to biofilm stained with $0.1 \%$ crystal violet and solubilised in $30 \%$ acetic acid at either 22 or $37^{\circ} \mathrm{C}$ two independent Pseudomonas aeruginosa PAO1 transposon insertion arsR mutants compared to WT. Error bars represent standard deviation. $n=4$ for each isolate/temperature condition. Details of the transposon mutants are available at:- http://www.pseudomonas.com/feature/show/?id= $107334 \&$ view=transposons.

\section{Additional file 2:}

Acknowledgements

We acknowledge the help of the great support provided by the technical team at MMU

\section{Authors' contributions}

MCE conceived the study. MCE JR, JW, AvB, MJ, EW, RR, JV, and PK helped design experimental procedures and contributed to the writing of the manuscript led by MCE, JR and AvB. Laboratory work was performed by JR and JW. The author(s) read and approved the final manuscript.

\section{Funding}

This work received no specific grant from any funding agency.

\section{Availability of data and materials}

Genbank accession numbers for all genome assembly sequences are shown in Suppl. Table 1. Phenotypic data for temperature-dependant biofilm analysis and stainless - steel biofilm analysis are shown in Suppl. Tables 1 and 2 respectively.

\section{Declarations}

Ethics approval and consent to participate

Not applicable.

\section{Consent for publication}

Not applicable.

\section{Competing interests}

The authors have no competing interests to declare.

\section{Author details}

${ }^{1}$ Faculty of Science and Engineering, Manchester Metropolitan University, Chester Street, Manchester, United Kingdom. ${ }^{2}$ bioMérieux SA, La Balme les Grottes, France.

Received: 30 October 2020 Accepted: 14 June 2021

Published online: 26 July 2021

\section{References}

1. Silby MW, et al. Pseudomonas genomes: diverse and adaptable. FEMS Microbiol Rev. 2011:35(4):652-80.

2. Boucher HW, et al. Bad bugs, no drugs: no ESKAPE! An update from the Infectious Diseases Society of America. Clin Infect Dis. 2009;48(1):1-12.

3. CDC UD.o.H.a.S.S., Antibiotic Resistant Threats in the United States 2019. 2019.

4. Tacconelli E, et al. Discovery, research, and development of new antibiotics: the WHO priority list of antibiotic-resistant bacteria and tuberculosis. Lancet Infect Dis. 2018;18(3):318-27.

5. Curran B, et al. Development of a multilocus sequence typing scheme for the opportunistic pathogen Pseudomonas aeruginosa. J Clin Microbiol. 2004;42(12):5644-9.

6. van Belkum A, et al. Phylogenetic Distribution of CRISPR-Cas Systems in Antibiotic-Resistant Pseudomonas aeruginosa. MBio. 2015;6(6):e01796-15.

7. Jaillard $\mathrm{M}$, et al. Correlation between phenotypic antibiotic susceptibility and the resistome in Pseudomonas aeruginosa. Int J Antimicrob Agents. 2017:50(2):210-8.

8. Kos VN, et al. The resistome of Pseudomonas aeruginosa in relationship to phenotypic susceptibility. Antimicrob Agents Chemother. 2015;59(1):427-36

9. Turton JF, et al. High-Resolution Analysis by Whole-Genome Sequencing of an International Lineage (Sequence Type 111) of Pseudomonas aeruginosa Associated with Metallo-Carbapenemases in the United Kingdom. J Clin Microbiol. 2015;53(8):2622-31.

10. Pirnay J-P, et al. Pseudomonas aeruginosa Population Structure Revisited. PLOS ONE. 2009;4(11):e7740. 
11. Oliver A, et al., The increasing threat of Pseudomonas aeruginosa high-risk clones. Drug Resistance Updates, 2015. 21-22(Supplement C): p. 41-59.

12. Sánchez-Diener I, et al., Interplay among Resistance Profiles, High-Risk Clones, and Virulence in the Caenorhabditis elegans Pseudomonas aeruginosa Infection Model. Antimicrobial Agents and Chemotherapy, 2017. 61(12).

13. Rasamiravaka T, et al., The Formation of Biofilms by Pseudomonas aeruginosa: A Review of the Natural and Synthetic Compounds Interfering with Control Mechanisms. BioMed Research International, 2015. 2015: p. 17.

14. Hutchison ML, Govan JRW. Pathogenicity of microbes associated with cystic fibrosis. Microbes Infect. 1999;1(12):1005-14.

15. Wendel AF, et al. Species Diversity of Environmental GIM-1-Producing Bacteria Collected during a Long-Term Outbreak. Appl Environ Microbiol. 2016;82(12):3605-10

16. Verran J, Redfern J. Testing Surface cleanability in Food Processing, Handbook of Hygiene Control in the Food Industry, Huub Lelieveld, John Holah, and D. Gabrić, Editors. 2016, Woodhead Publishing Limited: Cambridge. pp. 651-9.

17. Salm F, et al. Prolonged outbreak of clonal MDR Pseudomonas aeruginosa on an intensive care unit: contaminated sinks and contamination of ultrafiltrate bags as possible route of transmission? Antimicrobial Resistance Infection Control. 2016;5(1):53.

18. Mulet X, et al. Biological Markers of Pseudomonas aeruginosa Epidemic High-Risk Clones. Antimicrob Agents Chemother. 2013:57(11):5527-35.

19. Varin $\mathrm{A}$, et al. High prevalence and moderate diversity of Pseudomonas aeruginosa in the U-bends of high-risk units in hospital. Int J Hyg Environ Health. 2017;220(5):880-5.

20. A Bundle of Measures to Control an Outbreak of Pseudomonas aeruginosa Associated With P-Trap Contamination. Infection Control \& Hospital Epidemiology, 2018. 39(2): p. 164-169.

21. Scotland HP. Summary of Incident and Findings of the NHS Greater Glasgow and Clyde: Queen Elizabeth University Hospital/Royal Hospital for Children water contamination incident and recommendations for NHSScotland. Available from: https://www.hps. scot.nhs.uk/web-resources-container/summary-of-incident-and-findingsof-the-nhs-greater-glasgow-and-clyde-queen-elizabeth-universityhospitalroyal-hospital-for-children-water-contamination-incident-andrecommendations-for-nhsscotland/. 2019.

22. Aanensen DM, et al., Whole-Genome Sequencing for Routine Pathogen Surveillance in Public Health: a Population Snapshot of Invasive Staphylococcus aureus in Europe. mBio, 2016. 7(3).

23. Quainoo S, et al. Whole-Genome Sequencing of Bacterial Pathogens: the Future of Nosocomial Outbreak Analysis. Clin Microbiol Rev. 2017;30(4): 1015-63.

24. Chen PE, Shapiro BJ. The advent of genome-wide association studies for bacteria. Curr Opin Microbiol. 2015;25:17-24.

25. Read TD, Massey RC. Characterizing the genetic basis of bacterial phenotypes using genome-wide association studies: a new direction for bacteriology. Genome medicine. 2014;6(11):109-9.

26. Gardner SN, Slezak T, Hall BG. KSNP3.0: SNP detection and phylogenetic analysis of genomes without genome alignment or reference genome. Bioinformatics. 2015:31(17):2877-8.

27. Mac Aogain M, et al. The non-classical ArsR-family repressor PyeR (PA4354) modulates biofilm formation in Pseudomonas aeruginosa. Microbiology. 2012;158(Pt 10):2598-609.

28. Andres J, Bertin PN. The microbial genomics of arsenic. FEMS Microbiol Rev. 2016;40(2):299-322.

29. Marchal M, et al. Effect of arsenite on swimming motility delays surface colonization in Herminiimonas arsenicoxydans. Microbiology. 2010;156(8): 2336-42.

30. OToole GA, Kolter R. Flagellar and twitching motility are necessary for Pseudomonas aeruginosa biofilm development. Mol Microbiol. 1998;30(2): 295-304.

31. Hockett $\mathrm{KL}$, Burch $\mathrm{AY}$, Lindow SE. Thermo-regulation of genes mediating motility and plant interactions in Pseudomonas syringae. PLoS One. 2013; 8(3):e59850.

32. McGroarty EJ, Koffler H, Smith RW. Regulation of flagellar morphogenesis by temperature: involvement of the bacterial cell surface in the synthesis of flagellin and the flagellum. J Bacteriol. 1973;113(1):295-303.

33. Peel M, Donachie W, Shaw A. Temperature-dependent expression of flagella of Listeria monocytogenes studied by electron microscopy, SDS-PAGE and western blotting. J Gen Microbiol. 1988;134(8):2171-8.
34. Nepper JF, Lin YC, Weibel DB. Rcs Phosphorelay Activation in CardiolipinDeficient Escherichia coli Reduces Biofilm Formation. J Bacteriol, 2019. 201(9).

35. Schmidt J, et al. The Pseudomonas aeruginosa chemotaxis methyltransferase CheR1 impacts on bacterial surface sampling. PLoS One 2011;6(3):e18184.

36. Davies DG, et al. The involvement of cell-to-cell signals in the development of a bacterial biofilm. Science. 1998;280(5361):295-8.

37. Carver TJ, et al. ACT: the Artemis Comparison Tool. Bioinformatics. 2005; 21(16):3422-3.

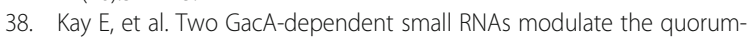
sensing response in Pseudomonas aeruginosa. J Bacteriol. 2006;188(16): 6026-33.

39. Cianciotto NP, White RC. Expanding Role of Type II Secretion in Bacterial Pathogenesis and Beyond. Infect Immun, 2017. 85(5).

40. Moradali MF, et al. Alginate Polymerization and Modification Are Linked in Pseudomonas aeruginosa. mBio. 2015;6(3):e00453-15.

41. Aldridge $P$, et al. Role of the GGDEF regulator PleD in polar development of Caulobacter crescentus. Mol Microbiol. 2003;47(6):1695-708.

42. Wei Q, et al., Diguanylate Cyclases and Phosphodiesterases Required for Basal-Level c-di-GMP in Pseudomonas aeruginosa as Revealed by Systematic Phylogenetic and Transcriptomic Analyses. Appl Environ Microbiol, 2019. 85(21)

43. Ruer $\mathrm{S}$, et al. Assembly of Fimbrial Structures in Pseudomonas aeruginosa: Functionality and Specificity of Chaperone-Usher Machineries. J Bacteriol. 2007;189(9):3547-55

44. Wen Y, Behiels E, Devreese B. Toxin-Antitoxin systems: their role in persistence, biofilm formation, and pathogenicity. Pathog Dis. 2014;70(3): 240-9.

45. Hengge R, et al. Bacterial Signal Transduction by Cyclic Di-GMP and Other Nucleotide Second Messengers. J Bacteriol. 2016;198(1):15-26.

46. Bouckaert R, et al. BEAST 2: A Software Platform for Bayesian Evolutionary Analysis. PLOS Computational Biology. 2014;10(4):e1003537.

47. Seemann T. Prokka: rapid prokaryotic genome annotation. Bioinformatics. 2014;30(14):2068-9.

48. Page AJ, et al. Roary: rapid large-scale prokaryote pan genome analysis Bioinformatics. 2015;31(22):3691-3.

49. Hadfield J, et al., Phandango: an interactive viewer for bacterial population genomics. Bioinformatics, 2017: p. btx610-btx610.

50. Coffey BM, Anderson GG. Biofilm Formation in the 96-Well Microtiter Plate, Pseudomonas Methods and Protocols, A. Filloux and J.-L. Ramos, Editors. 2014, Springer New York: New York, NY. pp. 631-41.

51. Kelly PJ, Arnell RD. Magnetron sputtering: a review of recent developments and applications. Vacuum. 2000;56(3):159-72.

52. Coenye T, et al. Should standardized susceptibility testing for microbial biofilms be introduced in clinical practice? Clin Microbiol Infect. 2018;24(6):570-2.

53. Laabei $M$, et al. Predicting the virulence of MRSA from its genome sequence. Genome Res. 2014;24(5):839-49.

54. Brynildsrud $\mathrm{O}$, et al. Rapid scoring of genes in microbial pan-genome-wide association studies with Scoary. Genome Biol. 2016;17(1):238.

55. Lees JA, et al. pyseer: a comprehensive tool for microbial pangenome-wide association studies. Bioinformatics. 2018;34(24):4310-2.

56. Benjamini Y, Hochberg Y. Controlling the False Discovery Rate: A Practical and Powerful Approach to Multiple Testing. J Roy Stat Soc B. 1995;57:289-300.

57. Bonferroni CE. /l calcolo delle assicurazioni su gruppi di teste, in Studi in Onore del Professore Salvatore Ortu Carboni. Italy: Rome; 1935. pp. 13-60.

58. Zimmermann L, et al., A Completely Reimplemented MPI Bioinformatics Toolkit with a New HHpred Server at its Core. Journal of Molecular Biology, 2017.

59. Stover CK, et al. Complete genome sequence of Pseudomonas aeruginosa PAO1, an opportunistic pathogen. Nature. 2000:406(6799):959-64.

60. Wang D, et al., Genome Sequence of Mucoid Pseudomonas aeruginosa Strain FRD1. Genome Announc, 2015. 3(2).

61. Miyoshi-Akiyama T, et al. Complete genome sequence of highly multidrugresistant Pseudomonas aeruginosa NCGM2.S1, a representative strain of a cluster endemic to Japan. J Bacteriol. 2011;193(24):7010.

62. Carver T, et al. Artemis: an integrated platform for visualization and analysis of high-throughput sequence-based experimental data. Bioinformatics. 2012;28(4):464-9.

\section{Publisher's Note}

Springer Nature remains neutral with regard to jurisdictional claims in published maps and institutional affiliations. 